\title{
Novel Hollow Core Fibers for Ultra-high Power Delivery
}

\author{
N. V. Wheeler*, Y. Chen, J. R. Hayes, T. D. Bradley, H. C. H. Mulvad, S. Abokhamis Mousavi, \\ S. R. Sandoghchi, M. A. Gouveia, E. Numkam, G. T. Jasion, M. B. S. Nawazuddin, P. Horak, \\ S. U. Alam, M. N. Petrovich, F. Poletti and D. J. Richardson. \\ Optoelectronics Research Centre, University of Southampton, Southampton, SO17 1BJ, U.K. \\ *Corresponding author: $\underline{\text { n.v.wheeler@soton.ac.uk }}$
}

\begin{abstract}
We review and compare recent hollow core photonic crystal fibers, both bandgap-guiding and antiresonant, which were designed and fabricated for high power laser delivery applications.
\end{abstract}

High power lasers are routinely used across a wide range of applications including welding, metal cutting and surgery. A wide range of laser sources are deployed and different parameters such as pulse length, beam quality and wavelength define their application space. Within these laser sources there are a growing range of ultra-high average power lasers, as well as ultra-short pulse lasers, which have a wide spectral bandwidth and a very high peak power. Ideally, the laser output will be delivered to the point of need via a flexible and versatile beam delivery system, which is able to maintain or even improve the beam quality, while transporting the laser light with minimal attenuation.

A fiber format is ideal for this and various demonstrations (e.g., $[1,2]$ ) have shown that hollow core photonic crystal fibers (HC-PCFs) provide significant advantages over more conventional solid core fibers because in a HC-PCF $>99 \%$ of the laser light is guided in air (or even in vacuum). This low overlap with the fiber cladding structure results in reduction of non-linearity by at least three orders of magnitude, as well as a substantial increase in the laser-induced damage threshold. Furthermore, HC-PCFs can be designed to operate over a wide range of transmission windows. In fact, again due to the low overlap between the guided light and the fiber cladding, the spectral transmission of a hollow core fiber can extend well beyond that of the material that it is fabricated from. For example, silica-based hollow core fibers can transmit light with sub $\mathrm{dB} / \mathrm{m}$ loss up to $\sim 4.5 \mu \mathrm{m}$ wavelength [3] even though the attenuation of silica rapidly increases beyond $2 \mu \mathrm{m}$.

Yet, the term hollow core photonic crystal fiber encompasses a wide range of fiber designs that can have vastly differing properties and therefore the right choice of fiber will be application dependent. In particular, HC-PCFs are generally classified into two distinct types, which are defined by their guidance mechanism: hollow core photonic bandgap fibers (HC-PBGFs) and anti-resonant/ inhibited coupling fibers (HC-ARFs). We have recently reported state-of-the art fibers of both types $[4,5,6]$ and in this work, we will review three fiber designs in detail which in this case are optimized for operation around the $1 \mu \mathrm{m}$ spectral region. Scanning electron microscope images and attenuation curves of examples of these fibers are shown in figure 1 . We will compare key performance parameters, such as attenuation, mode field diameter, field overlap with the cladding structure, bend sensitivity, modal content and dispersion. 
(a)

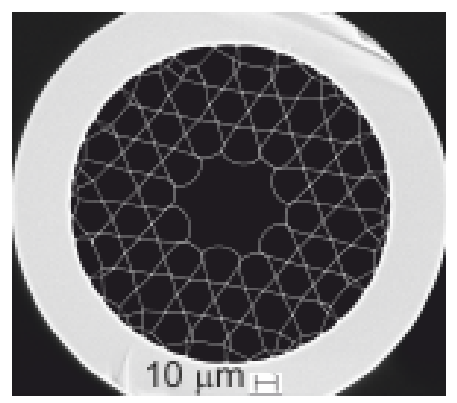

(b)

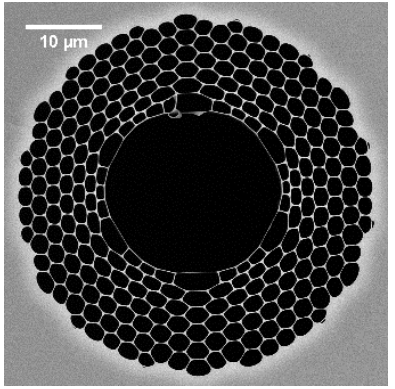

(c)

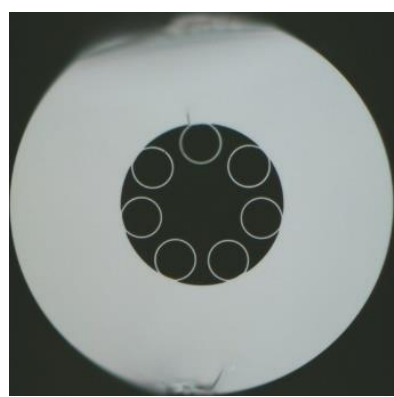

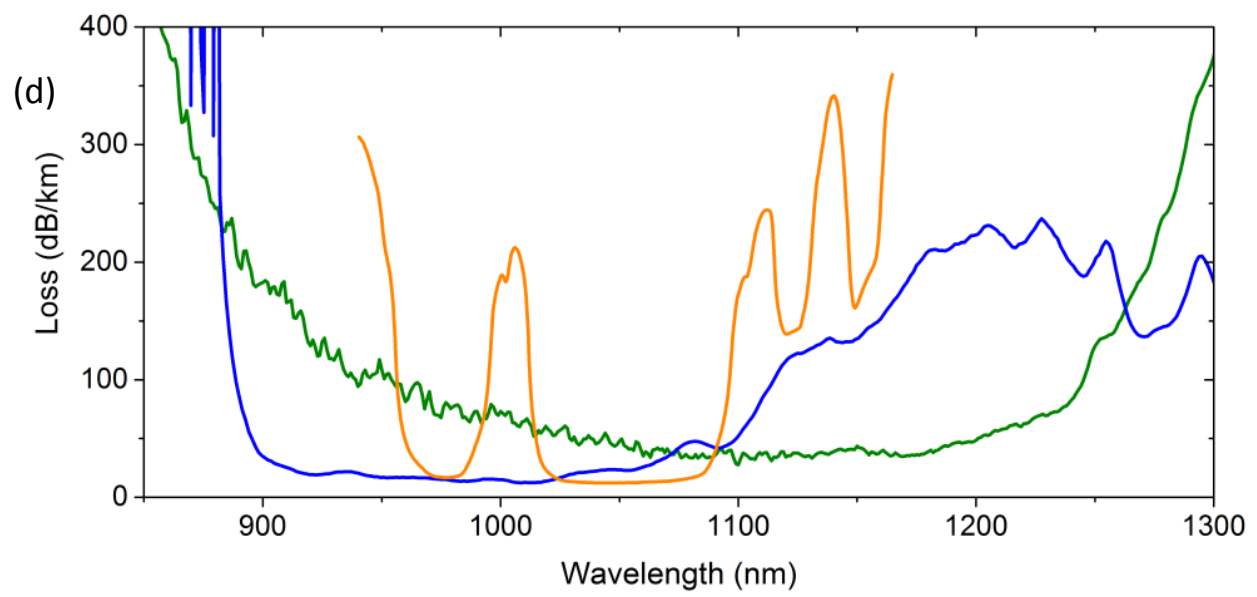

Figure 1: (a), (b) Scanning electron microscope images of state-of-the-art 7-cell Kagome hollow core fiber [4] and 37-cell hollow core photonic bandgap fiber [5] respectively; (c) Optical image of a tubular, non-contact hollow core fiber. All three fibers are designed for operation around $1 \mu \mathrm{m}$. (d) loss measurements for three examples of the fiber designs shown above (Kagome, blue line; 37 cell photonic bandgap fiber, orange line; tubular hollow core fiber, green line).

\section{References}

[1] C. Saraceno, F. Emaury, A. Diebold, I. Graumann, M. Golling, and U. Keller, "Trends in high-power ultrafast lasers," in SPIE Defense+ Security. International Society for Optics and Photonics, 2016, vol. 9835, pp. 98350X-1-98350X-7.

[2] B. Debord, M. Alharbi, L. Vincetti, A. Husakou, C. Fourcade-Dutin, C. Hoenninger, E. Mottay, F. Gerome and F. Benabid, "Multi-meter fiber-delivery and pulse self-compression of milli-Joule femtosecond laser and fiber-aided lasermicromachining.," Optics Express, vol. 22, no. 9, pp. 10735-46, 2014.

[3] F. Yu and J. C. Knight, "Spectral attenuation limits of silica hollow core negative curvature fiber," Optics Express 21(18) 21466, 2013.

[4] N. V. Wheeler, T.D. Bradley, J.R. Hayes, M.A. Gouveia, S. Liang, Y. Chen, S.R. Sandoghchi, S.M. Abokhamis Mousavi, F. Poletti, M.N. Petrovich and D.J. Richardson, "Low Loss Kagome Hollow Core Fibers Operating from the Near- to the MidIR," to be published in Optics Letters, 2017.

[5] Y. Chen, H.C.H. Mulvad, S. R. Sandoghchi, E. Numkam, T.D. Bradley, J. R. Hayes, N.V. Wheeler, G.T. Jasion, S. U. Alam, F. Poletti, M.N. Petrovich and D. J. Richardson, "First Demonstration of Low Loss, Bend Insensitive 37-Cell Hollow-Core Photonic Bandgap Fiber at $1 \mu \mathrm{m}$ for High Power Delivery Applications," Conference on Lasers Electro-Optics (CLEO) 2016, STu4P.1, 2016.

[6] J. R. Hayes, S. R. Sandoghchi, T. D. Bradley, Z. Liu, R. Slavík, M. A. Gouveia, N. V. Wheeler, G. Jasion, Y. Chen, E. Numkam Fokoua, M. N. Petrovich, D. J. Richardson, and F. Poletti "Antiresonant Hollow Core Fiber with Octave Spanning Bandwidth for Short Haul Data Communications," J. Light. Technol., 35(3) 437, 2017. 